\title{
EFFECT OF PARTICLE SIZE AND PACKING RATIO OF PID ON VIBRATION AMPLITUDE OF BEAM
}

\author{
P.S. Kachare ${ }^{1}$ and Bimleshkumar ${ }^{2}$ \\ ${ }^{1}$ Faculty of Mechanical Engineering Department \\ SVERI's College of Engineering, Pandharpur. \\ Gopalpur, Ranjani Road, Gopalpur, Pandharpur, \\ Solapur, Maharashtra, 413 304, INDIA \\ Email: kachareps@gmail.com; pravin_sk1@ rediffmail.com \\ ${ }^{2}$ Faculty of Mechanical Engineering Department \\ J. T. Mahajan College of Engineering, Faizpur. \\ Jivram Nagar, Faizpur, Yawal, Jalgaon, Maharashtra, 425 524, INDIA
}

\begin{abstract}
Everything in the universe that has mass possesses stiffness and intrinsic damping. Owing to the stiffness property, mass will vibrate when excited and its intrinsic damping property will act to stop the vibration. The particle impact damper (PID) is a very interesting damper that affects impact and friction effects of particles by means of energy dissipation. PID is a means for achieving high structural damping by using a particle-filled enclosure attached to a structure. The particles absorb the kinetic energy of the structure and convert it into heat through inelastic collisions between the particles themselves and between the particles and the walls of the enclosure. In this work, PID is measured for a cantilever mild steel beam with an enclosure attached to its free end; copper particles are used in this study. The PID is found to be highly nonlinear. The most useful observation is that for a very small weight penalty (about $7 \%$ to $8 \%$ ), the maximum damped amplitude of vibration at resonance with a PID, is about 9 to 10 times smaller than that without a PID. It is for more than that of with only intrinsic material damping of a majority of structural metals. A satisfactory comparison of damping with and without particles through experimentation is observed. The effect of the size of the particles on the damping performance of the beam and the effective packing ratio can be identified. It is also shown that as the packing ratio changes, the contributions of the phenomena of impact and friction towards damping also change. It is encouraging that despite its deceptive simplicity, the model captures the essential physics of PID.
\end{abstract}

Keywords: Particle; impact; friction; damping; vibration.

\section{INTRODUCTION}

A particle impact damper (PID) is an enclosure partially filled with particles. When the structure vibrates, particles in the cavity collide with each other and with the enclosure boundaries, causing damping through inelastic or nearly inelastic collisions. Such additional damping and mass modify the dynamic response of the primary system. The use of damping to control structural vibrations has been well documented (Beranek, 1971). Damping treatments are particularly useful for cases of harmonic excitation resulting in resonant or near-resonant response, broadband excitation resulting in a response dominated by resonant modes, and impact-excited free vibration resulting in 
undesirable and detrimental transient response. Damping treatments are used for noise reduction, fatigue reduction, vibration isolation, and absorption of impact energies. Traditional damping treatments use viscoelastic materials to convert strain energy into heat energy through the internal movements of long, intertwined molecules. Their primary disadvantage is that their properties depend on temperature, frequency, and strain; thus, they are difficult to use in environments with extreme temperatures or temperature gradients (Bell \& Bell, 1994). The earliest known work on the subject deals exclusively with impact dampers. An impact damper was studied for controlling aircraft flutter, fatigue, and vibration. Researchers assumed that two impacts occurred per cycle and they found that the maximum damping occurred if the phase angle between the motion of the auxiliary and primary mass was $180^{\circ}$ (Lieber \& Jensen, 1944). Two solutions for the motion of an impact damper were derived. The first describes the motions of the auxiliary and primary mass completely, which must be solved numerically after the first impact. The second solution assumes steady-state motion and is not complete. However, the two solutions agree with each other for a sufficiently large number of collisions. It was also found that the impact damper is most effective at resonance, and that by increasing the coefficient of restitution at resonance makes the damper more efficient. No experiments were performed to verify the solutions (Gurubin, 1956). Grubin's work was followed by an alternative method of obtaining a steady-state solution for the impact damper. This was done by modifying the disturbing force to have a phase component, which allows an impact at the right end of the container to occur at $t=0$. Both Warburton and Grubin assumed that two impacts occurred per cycle; an assumption that was later found true only under special circumstances (Warburton, 1957). One used three different materials to coat the impacting plates in their impact dampers. The three materials were aluminum, nylon, and rubber-coated aluminum. The enclosure was excited with a sine wave and it was found that the softer materials had a lower coefficient of restitution and provided a smaller amount of damping at resonance (Valuswami \& Crossley, 1975). The effects of gravity on impact dampers were examined and it was found that the dampers performed best under "zero gravity" conditions, i.e., when the damper was excited in a direction perpendicular to action of gravity. The dampers are most effective when two symmetric and equal impacts occurred per cycle and the effect of gravity caused the impulses at the ends of the container to be of unequal magnitude. The effect of gravity can be overcome if the acceleration of the enclosure is sufficiently high. This can be accomplished either by increasing the magnitude of the excitation force or by increasing the frequency of excitation (Sadek \& Williams, 1970). An impact damper was attached to the end of a cantilever beam and studied for both the transient-free decay and the steady-state forced vibration response. The transient-free decay showed an initial linear decay of vibrations, followed by an exponential decay after a considerable decrease in vibration. The linear decay corresponds to the damping by the impact damper, and the exponential decay corresponds to the damping inherent to the beam. This also shows that the impact damper ceases to work once acceleration amplitudes fall below a certain level. Forced vibration tests revealed an optimum gap size, which was a function of displacement amplitude and frequency. The gap size is simply the distance between the top of the particle bed and the ceiling of the enclosure (Bapat \& Sankar, 1985). These same results were later confirmed independently in an experiment with an impact damper attached to a leaf spring with a mass (Ema \& Marui, 1994). Eight different types of enclosure were investigated, including plastic bags, metal boxes, plastic boxes, and bags with an internal framework. It was found that the most effective enclosure was a hard plastic 
bag, which provided a coefficient of restitution near zero, quiet operation, and the second-highest loss factor. The metal box provided the highest loss factor, but it was accompanied by a high noise level and great sensitivity to the amount of clearance given to the particles. The response of the hard plastic bag was largely insensitive to gap size (Cempel \& Lotz, 1993; Mitra, Sahoo, \& Saha, 2012). Sensitivity to clearance was also investigated while studying different combinations of particle sizes. It was found that using a greater number of smaller particles resulted in less sensitivity to clearance. Unfortunately, the smaller particles were also less effective. However, an optimum clearance was still found for all cases (Papalou \& Masri, 1998). A series of experiments was performed designed to identify those design parameters that were most important for maximum damping. Seven different particle types of differing materials, sizes, and shapes were tested. It was discovered that particle damping has strong dependence on excitation amplitude, clearance, particle size, attachment location, and mass. There was weak dependence on particle shape. The majority of experimental work involving PIDs has used harmonic structural excitation (Hollkamp \& Gordon, 1998). However, both performed tests using random structural excitation. Papalou and Masri (1996) mounted a particle damper on an equivalent single-degree-of-freedom system. The damper dimensions could be changed by using four adjustable brackets. The authors reported that the effect of the container's dimensions seemed noticeable only when the ratio of the particle mass to the primary system mass was high. They also reported that a low mass ratio is beneficial when the damper movement is low. This stands in stark contrast to many other authors who all reported that a high mass ratio is beneficial, regardless of the damper movement (Panossian, 1991; Papalou \& Masri, 1996). Some authors have investigated particle damping as a means of fatigue reduction. A cantilever beam with a particle-filled enclosure attached to its free end was used for experimentation, in which damping was considered in the horizontal plane (perpendicular to gravity). It was noticed that damping was dependent on the clearance between the particles and the enclosure. The characteristics of the particle damping in transient vibrations have been discovered and simulated. It has been established that particle damping is a combination of the impact and friction damping. Furthermore, it has been shown that the displacement amplitude in the particle damping decreases linearly until the free vibration of the structure reaches a level below which no impact occurs (Mao, Wang, $\mathrm{Xu}, \&$ Chen, 2004). Computer simulation results were presented and used to investigate the damping performance of a single particle vertical impact damper over a wide range of excitation frequencies and amplitudes, particle-to-structure mass ratios, lid clearance ratios, structural damping ratios, and coefficients of restitution. Measurements of the damping performance, particle flight times, and structure contact times were presented. The performance of both the structure's undamped natural frequency and the offresonant conditions were studied in depth. Maximum damping at a fixed oscillation frequency occurred at an optimal lid height that increased with increasing mass ratio and increasing structural damping ratio, but decreased with coefficient of restitution. The corresponding maximum degree of damping increased with increasing mass ratio and coefficient of restitution, but decreased with increasing structural damping ratio. Field plots of the damping ratio were also presented as functions of oscillation amplitude and frequency, to demonstrate the damper performance over a range of design parameters and operating conditions (Duncan, Wassgren, \& Krousgrill, 2005). The work involved multi-unit particle dampers, which are passive damping devices involving granular particles in some cavities of a primary system. The principle behind particle damping is the removal of vibratory energy through losses that occur during the 
impact of the granular particles. This paper presents the results of experimental and analytical studies of the performance of a multi-unit particle damper in a horizontally vibrating system. An analytical solution based on the discrete element method is presented. Comparison between the experimental and analytical results shows that accurate estimates of the rms response of a primary system can be obtained. It is shown that the response of the primary system depends on the number of cavities and the cavity dimensions (Saeki, 2005). In this paper, the effect of the size of the particles and the packing ratio of the PID on damping performance of the beam is studied. It is shown experimentally that the most effective size of particles is $1 \mathrm{~mm}$ for the given set up, and that the effective packing ratio changes as per the condition of location, amplitude, and frequency of excitation.

\section{EXPERIMENTAL DESIGN}

A method is developed to find the effective size of particles and the packing ratio for a PID. Four sizes of particles: 1, 2, and $3 \mathrm{~mm}$ in diameter, and dust of copper material were selected for the study and for every size, the packing ratio was changed from $0 \%$ to $100 \%$ in steps of $25 \%$. To find an effective size of particle and to study the effects of packing ratio on damping performance, a cantilever beam of mild steel was selected. The physical parameters of the beam were: $450 \times 41.5 \times 5.63 \mathrm{~mm}$, Young's modulus of $200 \mathrm{GPa}$, density of $7594 \mathrm{Kg} / \mathrm{m}^{3}$, and moment of inertia of $617.15 \times 10^{-12} \mathrm{~m}^{4}$. This beam was fixed to the M.S. frame of 50-mm width, which gave a free length of $400 \mathrm{~mm}$ available as the cantilever beam. Three enclosures of differing diameters and heights were made of aluminum. The most effective enclosure of the three was found for a particular amplitude of excitation and low frequency range. The internal diameter and maximum internal height, expressed in millimeters, were 52 and 35, respectively, and the mass of that enclosure was $134.2 \mathrm{~g}$. Copper particles were used for the study with an effective mass of $55 \mathrm{~g}$. The effective location was selected as the free end of the cantilever. The essential data required were achieved with the following formulae and for that, a mathematical beam model was formed.
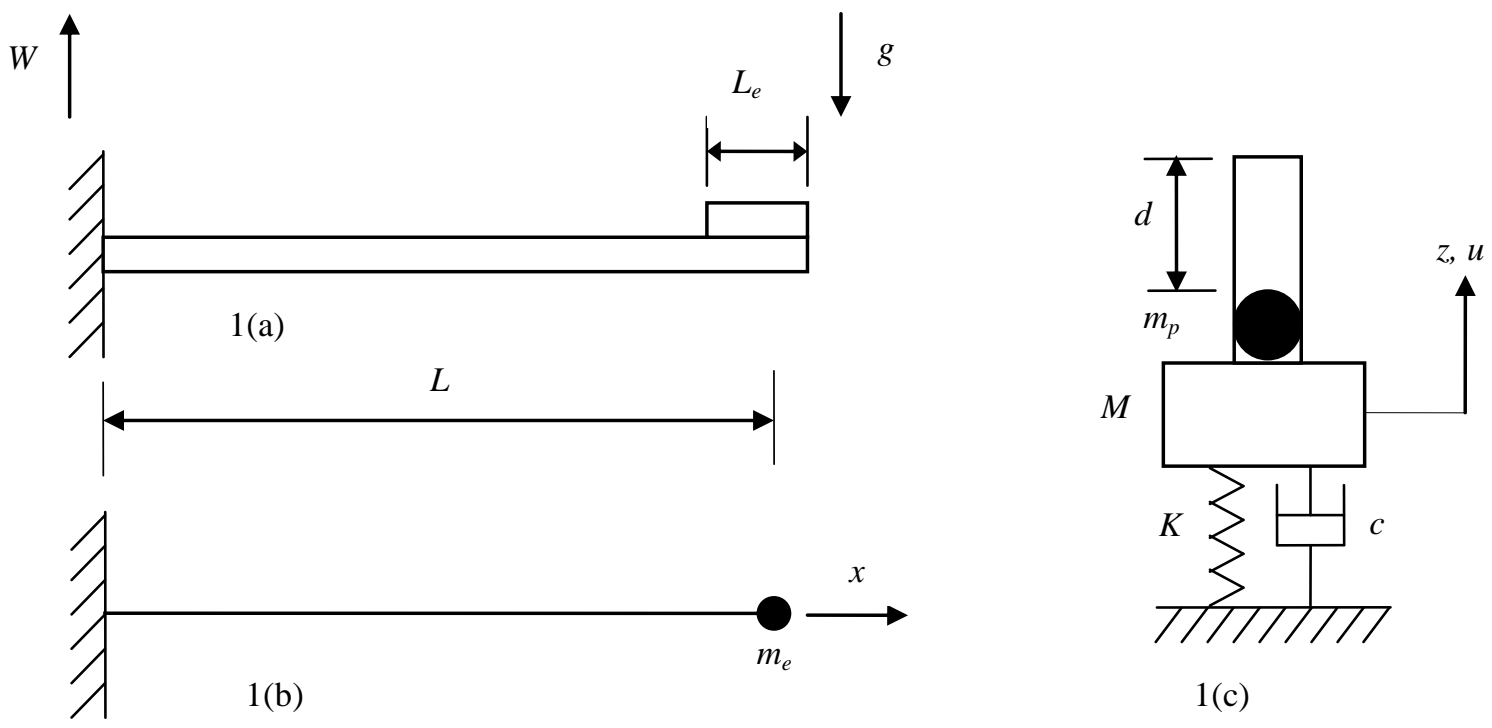

Figure 1. (a) Schematic of beam and enclosure, (b) model of beam with end mass $m_{e}$, (c) mathematical model equivalent to single degree of freedom. 
A cantilever beam, as shown in Figure 1(a), having length $L$ with known cross section was taken for study. The beam was idealized as a standard Euler-Bernoulli beam and the enclosure was assumed as a point mass attached to the end of beam, as shown in Figure 1(b). The length of the beam is along direction $x$ and it is a function of $x$. The single degree of freedom mathematical model of the beam is as shown in Figure1(c). The beam system is modeled as an effective mass $M$, effective stiffness $K$, and effective damping coefficient $c$. All the particles are assumed as an accumulated single mass $m_{p}$. The enclosure is a small tin in which the particles are placed, and it has a provision by which the clearance $d$ can be changed. The beam has constrained motion along the $z-$ direction and the instantaneous displacement $u$. The origin of coordinate $z$ is located at the equilibrium position of mass $M$ and the displacement $u$ of mass $M$ is measured from its equilibrium position with the particle mass $m_{p}$ resting at the bottom of the enclosure.

\section{Mathematical Expression}

The effective mass of the beam system $M$ is a function of the end mass and the reduced mass of the beam, and it is given as Eq. (1).

$$
M=m_{e}+m b_{\text {reduced }}
$$

in which end mass $m_{e}$ will have two different values as per the position of the particles in the enclosure:

$m_{e}=\left(m_{\text {encl }}+m_{p}\right)$ when the particles are moving in contact with the enclosure base,

$m_{e}=\left(m_{\text {encl }}\right)$ when the particles are moving separately from the enclosure.

$m_{\text {encl }}=$ mass of the enclosure.

$m b_{\text {reduced }}$ is a function of its length and is expressed with the help of Rayleigh's Energy method

$$
m b_{\text {reduced }}=\gamma \int_{0}^{L}[\phi(x)]^{2} d x
$$

To find $\phi(x)$, we consider a cantilever beam of mass $M_{b}$, having length $L$, and a point load of weight $W$ at the free end of the cantilever. We know that the maximum deflection of the cantilever is at the free end, which is given as

$$
Y_{\max }=-\frac{W L^{3}}{3 E I}
$$

The minus sign is because of the downward deflection.

In addition, the deflection of the cantilever beam, at any point on the beam at a distance $x$ from the fixed support, is

$$
y=\frac{1}{E I}\left[\frac{W x^{3}}{6}-\frac{W L x^{2}}{2}\right]
$$

Eq. (4) can be written in terms of $Y_{\max }$ as

$$
y=Y_{\max }\left[\frac{3 x^{2}}{2 L^{2}}-\frac{x^{3}}{2 L^{3}}\right]
$$

For the velocity of the beam, we differentiate Eq. (5) with respect to time $t$ 


$$
\frac{d y}{d t}=\dot{y}=\dot{Y}_{\max }\left[\frac{3 x^{2}}{2 L^{2}}-\frac{x^{3}}{2 L^{3}}\right]
$$

Invoking Rayleigh's maximum kinetic energy theorem, it can be written as:

$$
\begin{gathered}
\frac{1}{2} m v_{\max }^{2}=\frac{1}{2} m b_{\text {reduced }} v_{\max }^{2}=\frac{1}{2} \int_{0}^{L} \frac{M_{b}}{L}\left\{\dot{Y}_{\max }\left[\frac{3 x^{2}}{2 L^{2}}-\frac{x^{3}}{2 L^{3}}\right]\right\}^{2} d x \\
\frac{1}{2} m b_{\text {reduced }} v_{\text {max }}^{2}=\frac{1}{2} \gamma\left\{\int_{0}^{L}\left[\frac{3 x^{2}}{2 L^{2}}-\frac{x^{3}}{2 L^{3}}\right]^{2} d x\right\} \dot{Y}_{\max }^{2}
\end{gathered}
$$

From Eq. (8), $\phi(x)$ is given as

$$
\phi(x)=\left[\frac{3 x^{2}}{2 L^{2}}-\frac{x^{3}}{2 L^{3}}\right]
$$

where $x$ is the length of the beam to the point at which the effective mass is desired to be calculated. Accordingly, the limit of integration in Eq. (2) will change.

$$
M=m_{e}+\frac{M_{b}}{L} \int_{0}^{L}\left[\frac{3 x^{2}}{2 L^{2}}-\frac{x^{3}}{2 L^{3}}\right]^{2} d x
$$

The effective mass at the free end of the beam is calculated after solving Eq. (10) with an integration limit 0 to $L$

$$
M=m_{e}+0.2357 M_{b}
$$

From Eq. (11), it is clear that the effective mass of the beam $M$ at the free end, is the sum of the end mass and the reduced mass of the beam (reduced mass of the beam is $23.57 \%$ that of the actual mass of the beam). The reduced mass of the beam is 0.1673 $\mathrm{Kg}$.

$$
K=E I \int_{0}^{L}\left[\phi^{\prime \prime}(x)\right]^{2} d x
$$

It is observed that the reduced stiffness $K$ of beam is the same as that of the static stiffness of the beam (i.e., $3 E I / L^{3}$ ); for the test beam, it is $5.786 \mathrm{KN} \mathrm{m}^{-1}$.

The effective damping coefficient of the beam system $C$ is given as

$$
c=\frac{\psi_{b}}{2 \pi}(K / M)^{1 / 2}
$$

where $\Psi_{b}$ is the specific damping capacity of the beam material. The damping ratio can be given as

$$
\zeta=\left(c / c_{c r}\right)=\left(\frac{C}{2(K M)^{1 / 2}}\right)=\left(\psi_{b} / 4 \pi\right)
$$

This damping ratio $\zeta$ can be found by conducting a forced excitation test of the test beam within a frequency range of 0 to $70 \mathrm{~Hz}$ and then by using the Half Power Bandwidth method. The damping ratio $\zeta$ of the test beam is found to be 0.3 . The natural frequency of the beam without the enclosure is $\omega_{n}=\left(K / m b_{\text {reduced }}\right)^{1 / 2}=185.96$ $\mathrm{rad} / \mathrm{sec}$. Therefore, the natural frequency $f_{n}=(1 / 2 \pi)\left(K / m b_{\text {reduced }}\right)^{1 / 2}=29.61 \mathrm{~Hz}$, and the damped natural frequency of the beam is $f_{d}=\mathrm{f}_{\mathrm{n}}\left(1-\zeta^{2}\right)^{1 / 2}=28.24 \mathrm{~Hz}$. When the 
enclosure is attached at the free end of beam, then $\omega_{n}=(K / M)^{1 / 2}=138.53 \mathrm{rad} / \mathrm{sec}$ and therefore, the natural frequency $f_{n}=1 / 2 \pi(K / M)^{1 / 2}=22.05 \mathrm{~Hz}$ and the damped natural frequency is $f_{d=} f_{n}\left(1-\zeta^{2}\right)^{1 / 2}=21.03 \mathrm{~Hz}$.

The experimentation was done for both transient and forced excitation. Spherical particles of copper material of four different sizes were used: 1, 2, $3 \mathrm{~mm}$ in diameter, and dust. For each size, the packing ratio was changed from $0 \%$ to $100 \%$ in steps of $25 \%$. The readings were taken for $55 \mathrm{~g}$ of effective particle mass. The enclosure was fixed on the beam for transient excitation and an initial displacement of $18 \mathrm{~mm}$ was given to the beam at its free end. It was then allowed to vibrate freely and readings were taken on the FFT analyzer. Readings were also taken for forced excitation in the same way as discussed above. In this, the exciter tip was kept exactly below the beam's free end with minimal $(\approx 3 \mathrm{~mm})$ between them. This gap is kept constant for all the forced excitation readings. The enclosure was fixed on the free end of the beam by screws, and an accelerometer was attached below the beam near the point of excitation. The frequency of excitation was varied from 8 to $40 \mathrm{~Hz}$ in steps of $2 \mathrm{~Hz}$, which includes the resonance frequency of beam.

\section{RESULTS AND DISCUSSION}

\section{Transient Excitation}

Figures 2 and 3 explore the behavior of both the acceleration and displacement versus the packing ratio for the selected sizes of particles. The orientation of both graphs remains almost the same for all sizes of particles. The acceleration and displacement of the beam for all sizes of particles decreases linearly and with steeper gradient from $0 \%$ to $25 \%$ packing ratio. These quantities for $1-3-\mathrm{mm}$ particle sizes keep on reducing with a lessening gradient up to $50 \%$ and then they increase slowly up to $75 \%$ with the curves resembling a near-horizontal line. However, for dust, these quantities increase in the zone of $25 \%$ to $50 \%$ and $50 \%$ to $75 \%$ with an increasing slope. In the zone of $75 \%$ to $100 \%$, these quantities increase suddenly for all particle sizes including dust. This happens due to the proportion of the contribution of the impact and friction phenomena on the damping of the beam. From these graphs, it can be said that the PID is highly effective when the packing ratio is between $25 \%$ and $75 \%$. 


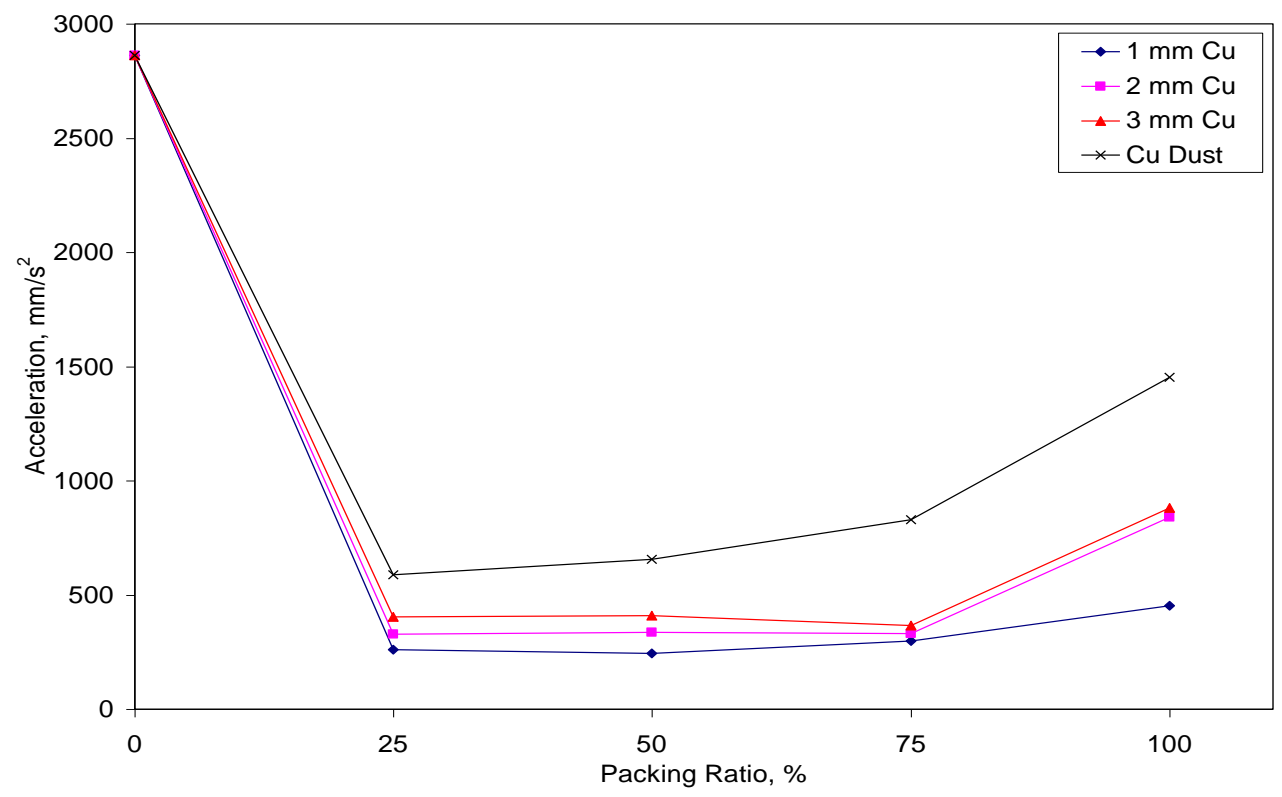

Figure 2. Acceleration versus packing ratio for transient vibration.

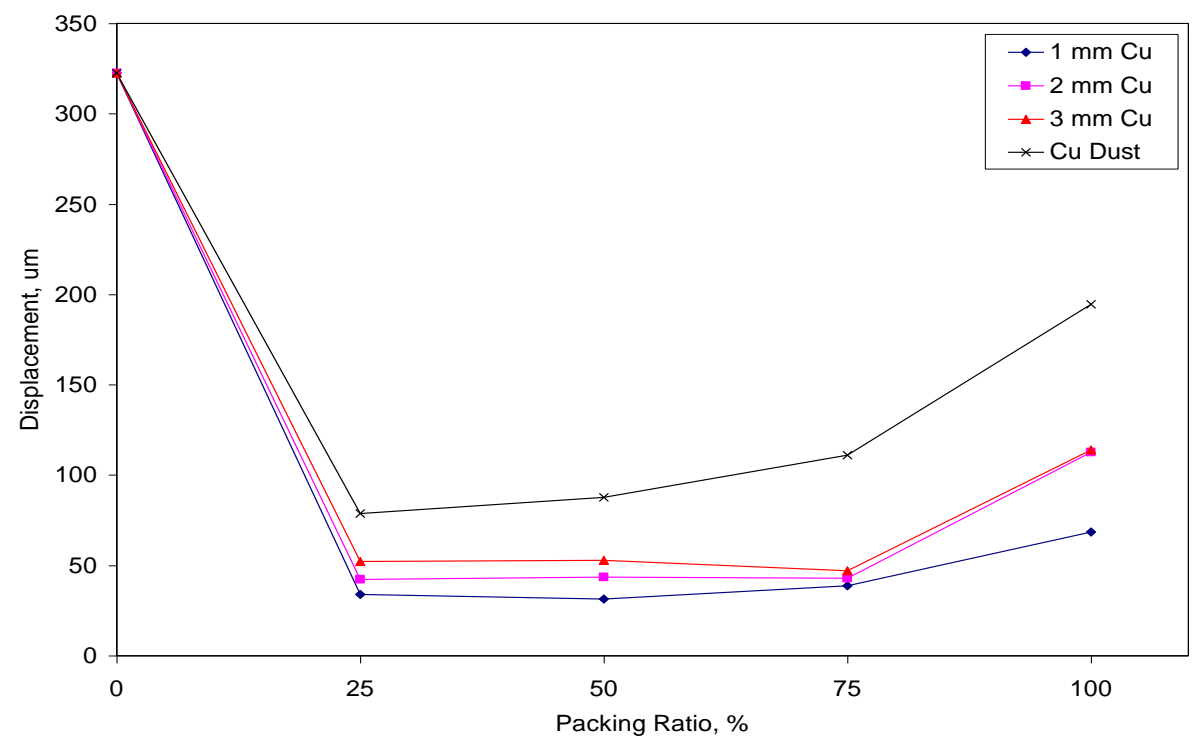

Figure 3. Displacement versus packing ratio for transient vibration.

The movement of particles in the enclosure (and hence, the number of impacts of the particles) is directly proportional to the displacement and acceleration obtained from the beam. Furthermore, as the size decreases, the number of particles for the said mass ratio increases and therefore, the probability of an impact increases. For biggersized particles, the beam acceleration is required to be greater, which will make the particles have impacts. It is observed that dust gives the minimum damping performance because there are no impacts and the lowest damping offered is due to friction. The 1-mm particles show the maximum damping performance. As the packing ratio increases (i.e., the number of particles close to each other increases), the friction effect increases, but simultaneously, the impact effect decreases. Hence, maximum damping can be achieved with particles of size $1 \mathrm{~mm}$ and packing ratio of $50 \%$. 


\section{Forced Excitation}

Figures 4-7 reveal the behavior of both acceleration and displacement versus excitation frequency for the different particle sizes and with five different packing ratios for every particle size. A $0 \%$ packing ratio means zero particles in the enclosure, which shows zero damping effect because there are no impacts and no friction. However, with packing ratios of $25 \%$ to $100 \%$, the variation in contribution of the impact and friction phenomena on damping can be seen easily. To have impacts of particles, the acceleration of the beam must be greater than the acceleration due to gravity and for this, the frequency range of 8 to $40 \mathrm{~Hz}$ was selected for the experimentation. This study was done with small amplitude and low frequency range of excitation. Figure 4 gives the damping performance of the beam with particles of size $1 \mathrm{~mm}$. It is found that the damping effect is highest at resonance with $25 \%$ packing ratio and that it decreases in a sequence of packing ratio from $25 \%-75 \%-50 \%-100 \%$, and this is true for almost all frequencies with few exceptions. This size and packing ratio of particles causes the impact phenomenon to dominate the friction phenomenon. In these graphs, it can also be seen that the effective packing ratio is between $25 \%$ and $75 \%$, and it is actually $25 \%$.

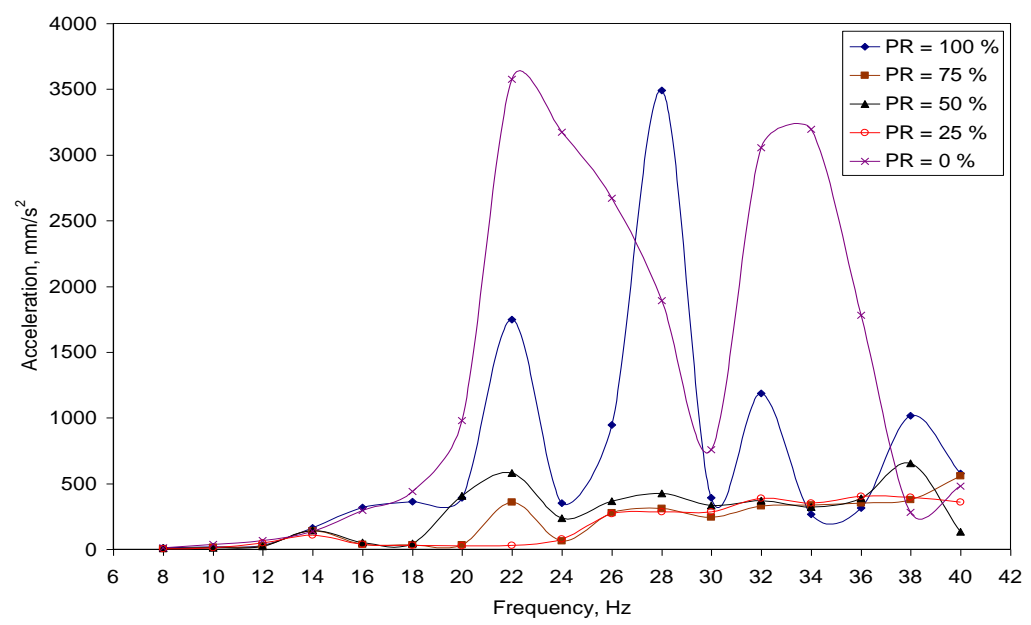

(a) Acceleration

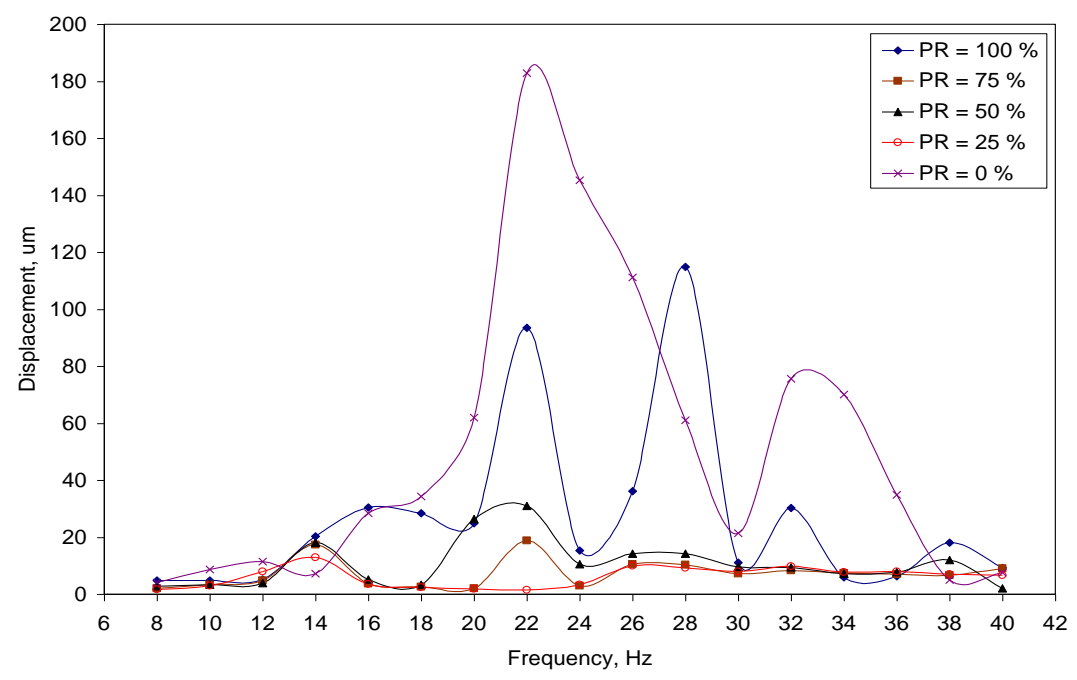

(b) Displacement

Figure 4. Variation of acceleration and displacement versus excitation frequency for forced vibration with 1-mm $\mathrm{Cu}$ balls. 
Figure 5 reveals the damping effect of the PID with 2-mm-sized particles. It is found that the highest damping effect is achieved with $100 \%$ packing ratio. In addition, the damping effect shown by packing ratios $25 \%$ and $50 \%$ are much closer to that of $100 \%$, and that the damping effect of $75 \%$ packing ratio is close to $0 \%$. Here, the friction phenomenon is strongest with $100 \%$ packing ratio and the impact phenomenon is strongest with $25 \%$ and $50 \%$ packing ratio. However, the $75 \%$ packing ratio does not give significant damping. Here, as size is increased, the surface area prone to friction is also increased and the frictional effect dominates the impact effect. Therefore, the effective damping is $100 \%$.

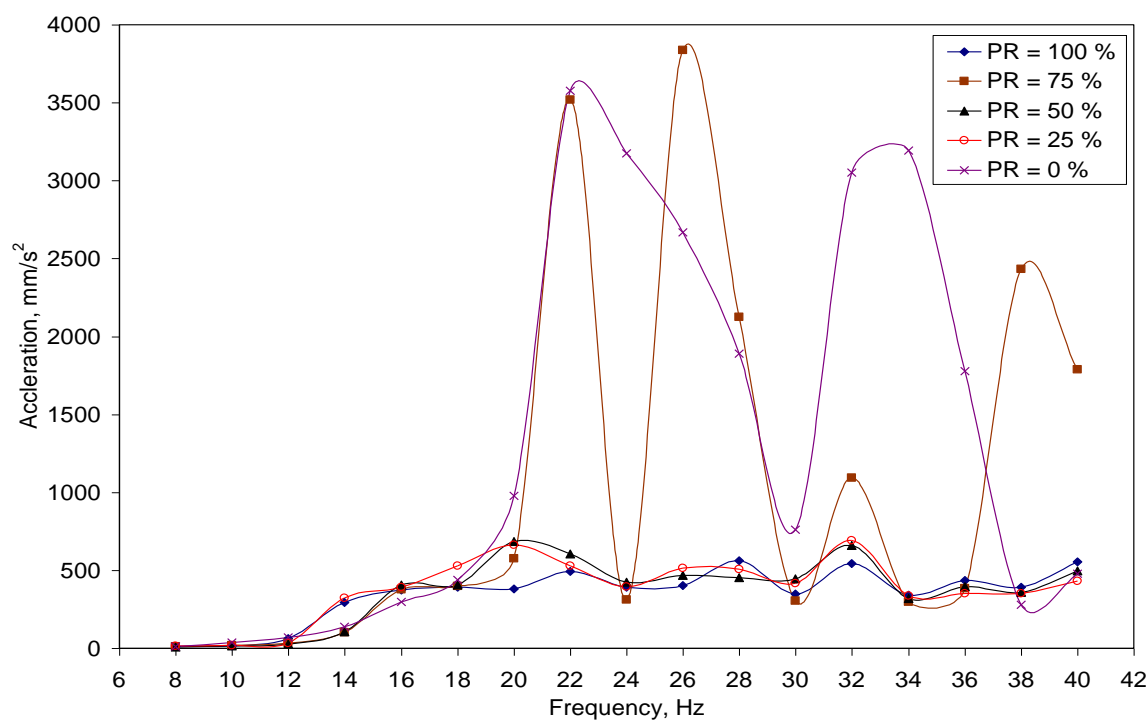

(a) Acceleration

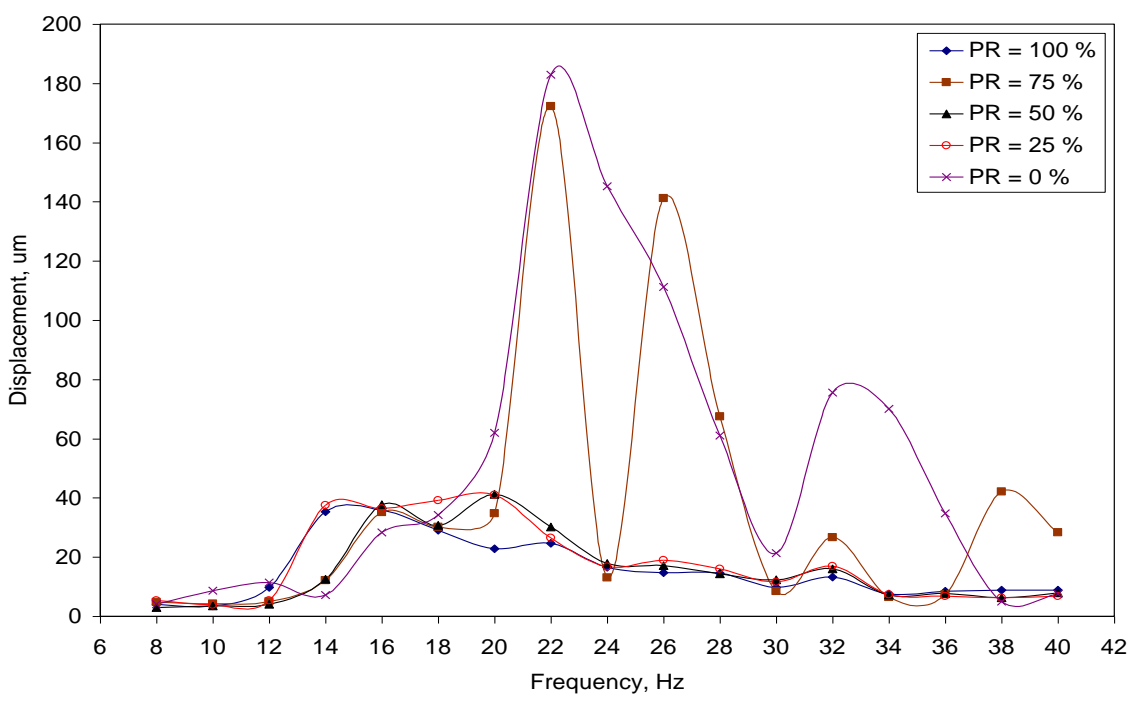

(b) Displacement

Figure 5. Variation of acceleration and displacement against excitation frequency for forced vibration with 2-mm Cu balls.

Figure 6 presents the damping performance of the beam with 3-mm-sized particles. Owing to the increased size, the mass of a single particle is larger and the excitation force falls short; thus, impacts are not be observed and the highest damping is due to 
friction with $100 \%$ packing ratio. In addition, other packing ratios show considerable damping. Here, it is also seen that as size is increased, the surface area prone to friction is also increased and that the frictional effect dominates the impact effect. Therefore, the effective damping is $100 \%$.

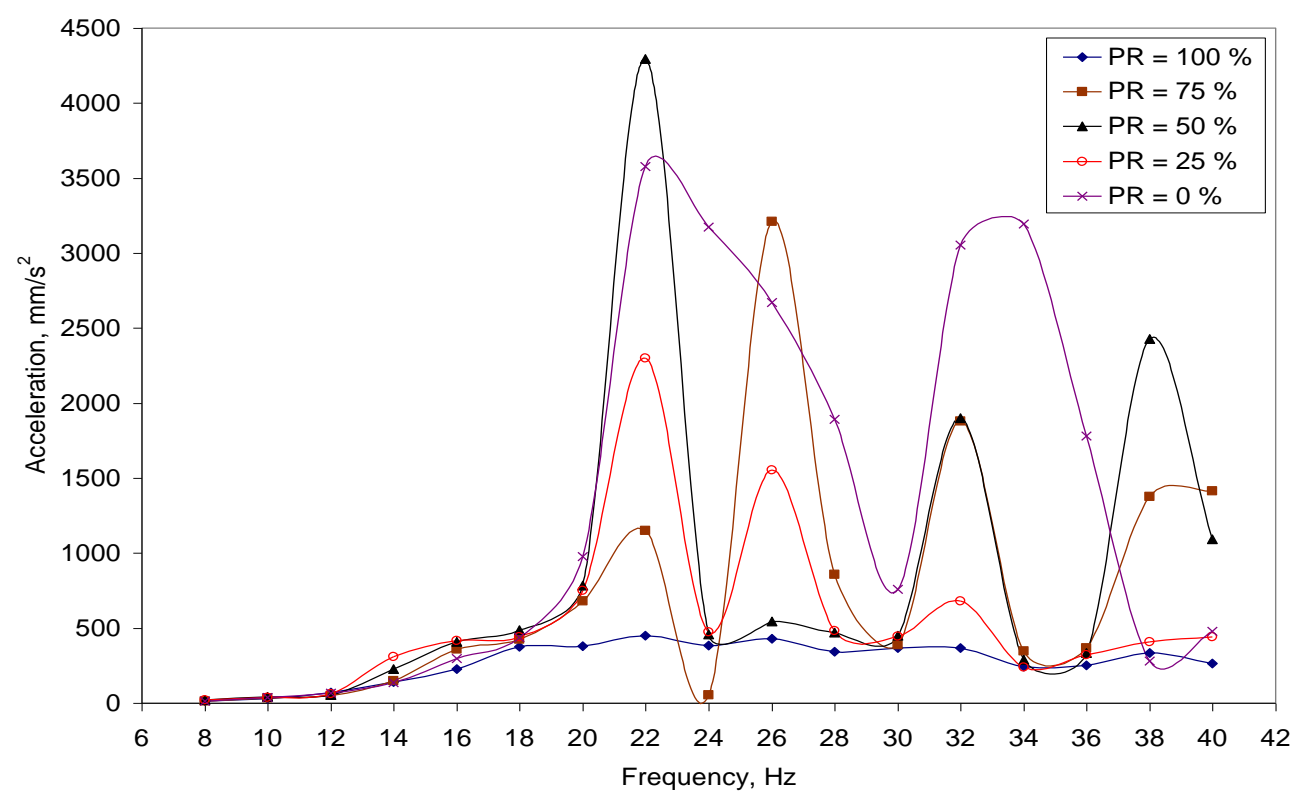

(a) Acceleration

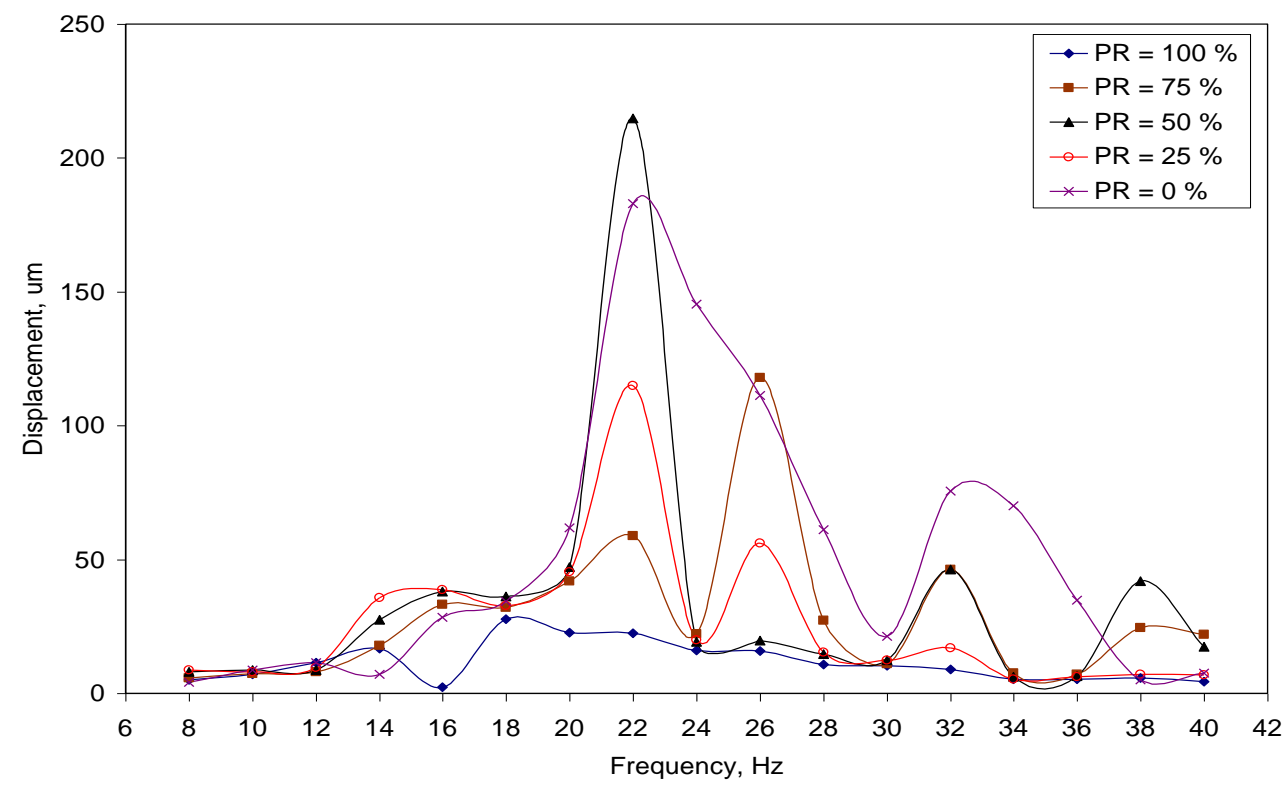

(b) Displacement

Figure 6. Variation of acceleration and displacement against excitation frequency for forced vibration with 3-mm Cu balls.

Figure 7 explores the dynamic response of the beam for copper dust. Under this condition, as the mass of a single particle is negligible, the observed damping is due to friction and not impact. In addition, the amount of damping is reduced compared with the other sizes and packing ratios. Therefore, it is better to avoid dust when considering PIDs. 


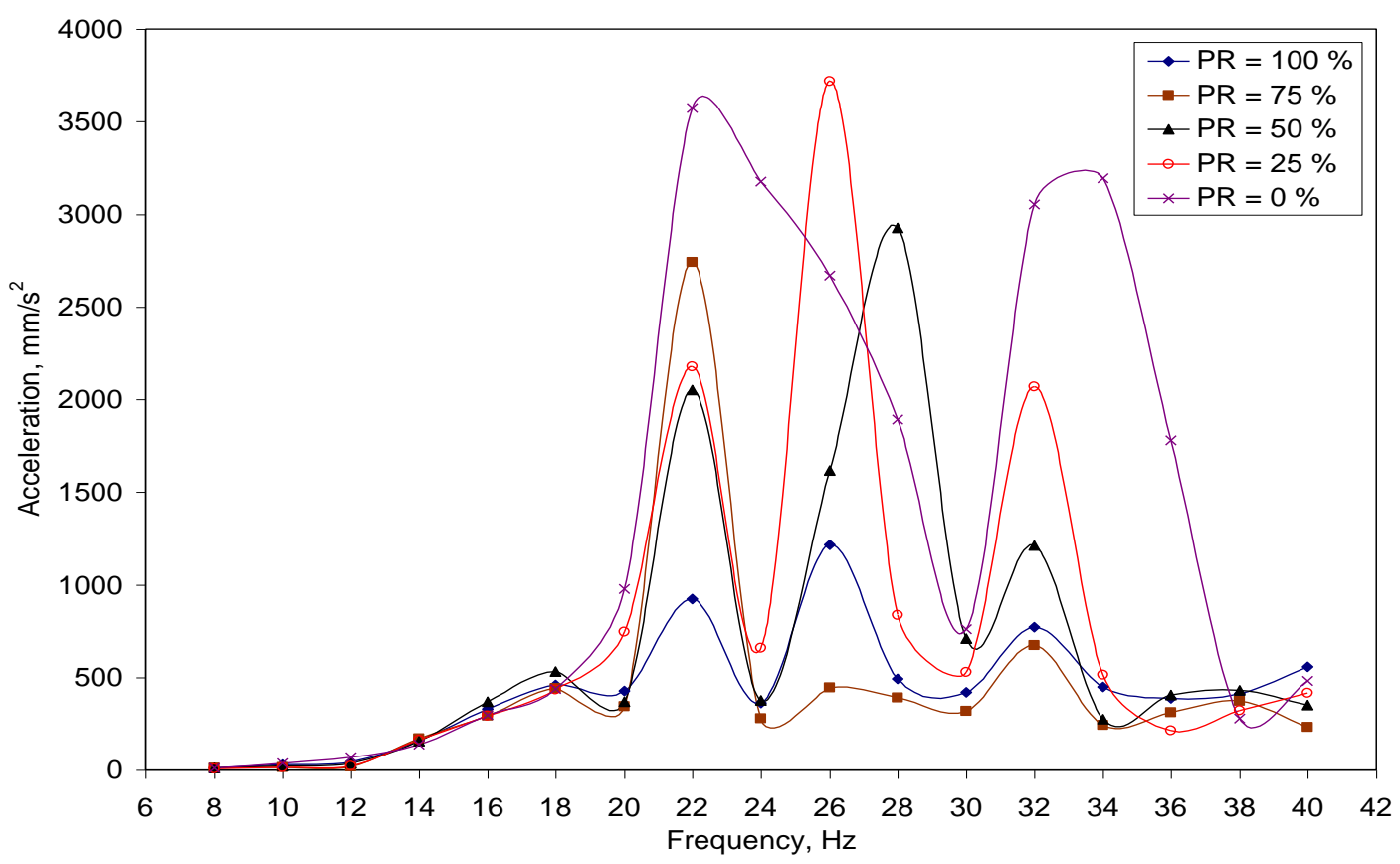

(a) Acceleration

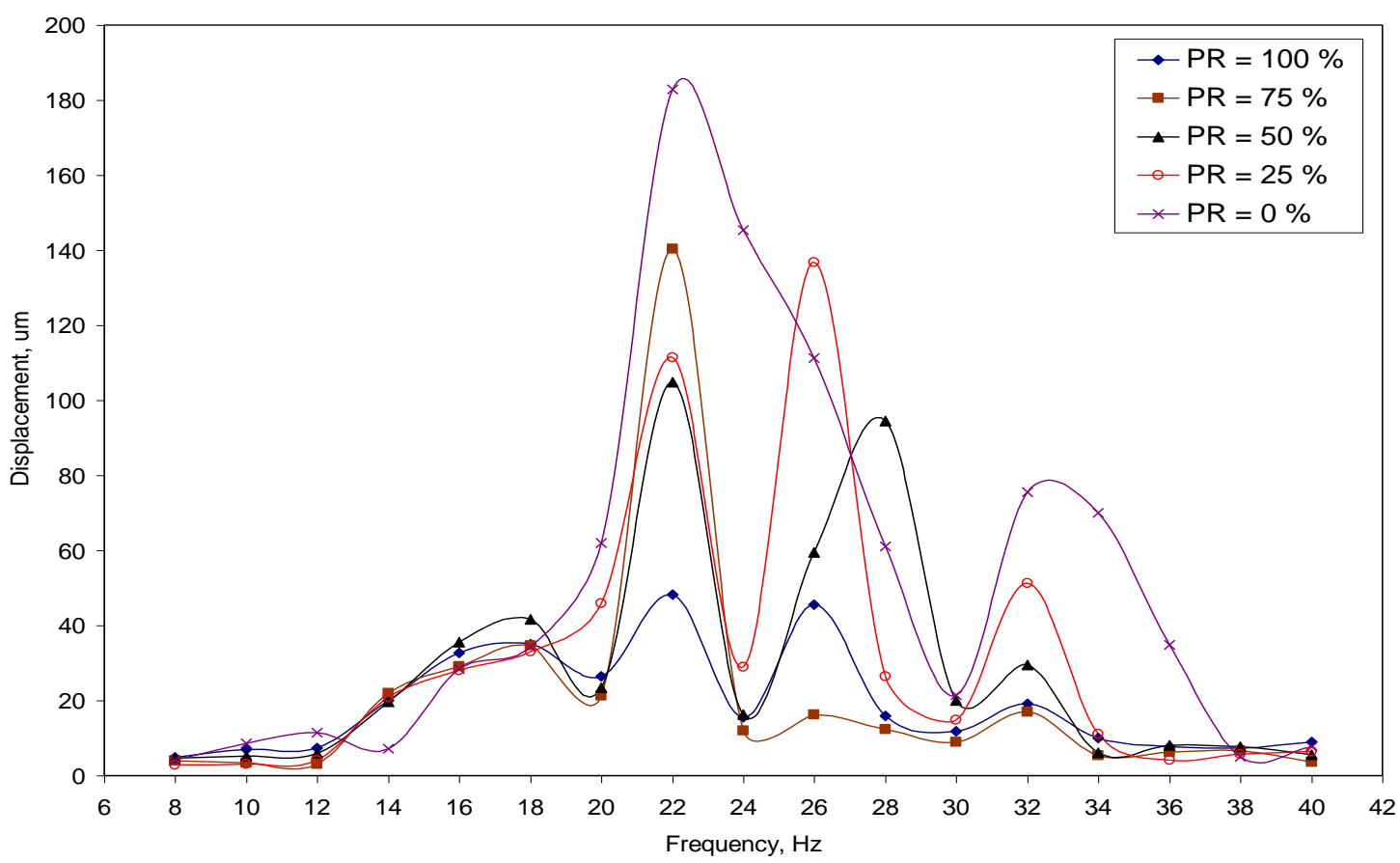

(b) Displacement

Figure 7. Variation of acceleration and displacement against excitation frequency for forced vibration with $\mathrm{Cu}$ dust.

\section{CONCLUSION}

It is observed that under both transient and forced vibration, the size of the particles of the PID is the key factor in achieving the maximum damping effect. In experimentation, 
it is observed that the 1-mm particles are the best size for the setup under consideration. It is shown that packing ratio also has an effect on damping. Under transient excitation, as the number of particles increases, the impact effect dominates the friction effect up to $50 \%$ packing ratio, beyond which the friction effect dominates the impact effect for all locations of the PID. However, the amount of damping due to the friction effect is smaller than that of the impact effect; this is due to the low frequency range. Under forced excitation, the effective packing ratio can be between $25 \%$ and $50 \%$ depending upon the excitation frequency and magnitude of the excitation force. Some nonlinearity in the behavior of the PID is observed.

\section{ACKNOWLEDGEMENTS}

The authors gratefully acknowledge the continuous support and encouragement given by Prof. B.P. Ronge, Secretary, SVERI's College of Eng., Pandharpur, and the timely guidance of Dr. S.G. Joshi, Ex. Professor and Head of Mech. Eng. Dept., W.C.E. Sangli. and Dr. Prashant Pawar, Coordinator, Research \& Development Cell, SVERI's College of Eng., Pandharpur.

\section{REFERENCES}

Bapat, C. N., \& Sankar, S. (1985). Single unit impact damper in free and forced vibration. Journal of Sound and Vibration, 99(1), 85-94.

Bell, L. H., \& Bell, D. H. (1994). Industrial noise control: fundamentals and applications. New York: Marcel Dekker, Inc.

Beranek, L. L. (1971). Noise and vibration control. New York: McGraw-Hill.

Cempel, C., \& Lotz, G. (1993). Efficiency of vibrational energy dissipation by moving shot. Journal of Structural Engineering, 119(9), 2642-2652.

Duncan, M. R., Wassgren, C. R., \& Krousgrill, C. M. (2005). The damping performance of a single particle impact damper. Journal of Sound and Vibration, 286, 123144.

Ema, S., \& Marui, E. (1994). A fundamental study on impact dampers. International Journal of Machine Tools and Manufacture, 34(3),407-421.

Grubin, C. (1956). On the theory of the acceleration damper. Transactions of ASME, Journal of Applied Mechanics, 23(3), 373-378.

Hollkamp, J. J., \& Gordon, R. W. (1998). Passive damping and isolation: experiments with particle damping. Proceedings of SPIE - the International Society for Optical Engineering. Smart Structures and Materials, pp. 2-12.

Lieber, P., \& Jensen, D. P. (1944). An acceleration damper: Development, design, and some applications. Transactions of the American Society of Mechanical Engineers, 67, 523-530.

Mao, K., Wang, M. Y., Xu, Z., \& Chen, T. (2004). Simulation and characterization of particle damping in transient vibrations. ASME Journal of Vibration and Acoustics, 126, 202-211.

Mitra, A., Sahoo, P., \& Saha, K. (2012). Large displacement of crossbeam structure through energy method. International Journal of Automotive and Mechanical Engineering, 5, 520-544.

Panossian, H. V. (1991). Non-obstructive particle damping tests on aluminum beams. Proceedings, Damping '91 Conference in San Diego, CA, pp. 13-15. 
Papalou, A., \& Masri, S. F. (1996). Response of impact dampers with granular materials under random excitation, Earthquake Engineering and Structural Dynamics, 25, 253-267.

Papalou, A., \& Masri, S. F. (1998). An experimental investigation of particle dampers under harmonic excitation. Journal of Vibration and Control, 4, 361-379.

Sadek, M. M., \& Williams, C. J. H. (1970). Effect of gravity on the performance of an impact damper: Part 2. Stability of vibrational modes. Journal of Mechanical Engineering Science, 12(4), 278-287.

Saeki, M. 2005, Analytical study of multi-particle damping. Journal of sound and vibration, 281, 1133-1144.

Valuswami, M. A., \& Crossley, F. R. E. (1975). Multiple impacts of a ball between two plates. Part 1: some experimental observations. Transactions of the American Society of Mechanical Engineers, Journal of Engineering for Industry, 97(3), 820-827.

Warburton, G. B. (1957). Discussion of on the theory of the acceleration damper, Transactions of ASME, Journal of Applied Mechanics, 24, 322-324. 